\title{
Narrativas autobiográficas, una estrategia pedagógica para transformar el ser desde la escritura*
}

Luz Marina Toro Toro

Institución Educativa Ángela Restrepo Moreno, Medellín, Colombia

Itoro6395@coredi.edu.co

\section{RESUMEN}

El presente artículo surge como producto de un proyecto de investigación, dirigido a analizar el papel que cumplen las narrativas autobiográficas para motivar las prácticas de escritura, y a su vez el conocimiento de sí mismo de los estudiantes del modelo "Caminar en secundaria". Se utilizó un enfoque cualitativo y un diseño micro etnográfico, basado en la observación e interpretación de las narrativas de los estudiantes, a fin de fortalecer los aspectos escriturales y el conocimiento de sí mismo. Como resultado se identificó en los estudiantes participantes un avance en sus prácticas de escritura, al contrastar los resultados de la prueba diagnóstica con la prueba final y una transformación en su ser al lograr escribir y leer sus historias. Se concluyó que los textos reflexivos que se produjeron, basados en las experiencias de vida de los estudiantes, son una estrategia para fortalecer las habilidades escriturales con este tipo de población extra edad, ya que a partir de sus necesidades e intereses se pueden formar estudiantes productores en lugar de reproductores de textos, pues la escritura está basada en experiencias reales que llevan a la reflexión y potencian su ser y su saber desde la escritura.

Palabras clave: pedagogía; narrativas autobiográficas; escritura reflexiva; sujeto.

Cómo citar: Toro Toro, L. M. (2019). Narrativas autobiográficas, una estrategia pedagógica para transformar el ser desde la escritura. Ciencias Sociales y Educación, 8(16), 91-112. DOI: https://doi.org/10.22395/csye.v8n16a6 Recibido: 14 de agosto de 2019.

Aprobado: 17 de noviembre de 2019. 


\section{Autobiographical Narratives, a Pedagogical Strategy for the Transformation of the Being from Writing}

\section{ABSTRACT}

This article is derived from a research project directed towards analyzing the role of the "Autobiographical analysis" for motivating writing practices and at the same time self-knowledge on students from the "Caminar en Secundaria" (Walking in High School) model. A qualitative model and a micro-ethnographic model were used, based on observation and interpretation of the student's narratives to strengthen the writing aspects and the self-knowledge. As a result, it was identified that participating students display advancement in their writing process when contrasted with the diagnosis and final tests and also a transformation in their beings by achieving writing and reading their stories. It was concluded that the reflective texts based on the life experiences of the students are a strategy for strengthening their writing abilities in this kind of extra-age population segment, given that based on their needs and interests they can become producers instead f reproducer of texts, given also that writing is based on real experiences that lead to reflection and enhances their being and their knowledge from writing.

Keywords: pedagogy; autobiographical narratives; reflective writing; subject.

\section{Narrativas autobiográficas: uma estratégia pedagógica para transformar o ser a partir da escrita}

\section{RESUMO}

Este artigo é produto de um projeto de pesquisa, dirigido à análise do papel que as narrativas autobiográficas cumprem para motivar as práticas de escrita e, por sua vez, o conhecimento de si mesmo dos estudantes do modelo "Caminhar em secundária". Foram utilizados abordagem qualitativa e desenho microetnográfico, com base na observação e interpretação das narrativas dos estudantes, a fim de fortalecer os aspectos escriturais e o conhecimento de si mesmo. Como resultado, foi identificado, nos estudantes, progresso em suas práticas de escrita, ao comparar os resultados do teste diagnóstico com o teste final e uma transformação em seu ser ao conseguir escrever e ler suas histórias. Conclui-se que os textos de reflexão produzidos, baseados nas experiências dos estudantes, são uma estratégia para fortalecer as habilidades de escrita com esse tipo de população extraidade, já que, com base em suas necessidades e interesses, podem ser formados estudantes produtores em lugar de reprodutores de textos, pois a escrita está baseada em experiências reais que levam à reflexão e potencializam seu ser e seu saber a partir da escrita.

Palavras-chave: pedagogia; narrativas autobiográficas; escrita reflexiva; sujeito. 


\section{Introducción}

Este artículo surge como producto de la investigación Narrativas autobiográficas una propuesta pedagógica para crear y recrear las prácticas de escritura: me leo, me pienso, me escribo, me transformo y aprendo. La situación problema fue identificada en la institución educativa Ángela Restrepo Moreno del Municipio de Medellín donde está presente el modelo "Caminar en secundaria", creado por el gobierno para garantizar el derecho a la educación de una población que por diferentes motivos como el desplazamiento forzado, problemas económicos, pérdidas de años anteriores por desmotivación o situaciones familiares, se encuentran en grado sexto y en condición extra edad. El programa busca que aquellos jóvenes que deseen continuar en el sistema educativo, se escolaricen y puedan nivelarse académicamente. La idea es que en un acelerado proceso académico se cursen dos grados en un año para prepararse a iniciar su grado octavo, sin tener en cuenta que las prácticas de escritura son deficientes, no solo por el poco tiempo para el proceso académico, sino por la apatía que presentan los jóvenes a nivel general a las practicas escriturales. En la Institución Educativa Ángela Restrepo Moreno se cuenta con dos grupos de esta población, uno de ellos es el grado sexto cinco conformado por veinticinco estudiantes, quienes presentan deficiencias en sus prácticas de escritura tales como: parafraseo de textos sin comprensión alguna, déficit de bagaje verbal, producción textual incoherente e inconsistente, reproducción de textos a través de libros, celulares y cuadernos de forma superficial, sin realizar un proceso de interiorización, todo esto sumado a la apatía por lo académico, debido a los paradigmas y las emociones reprimidas frente a las problemáticas sociales y familiares que han marcado su vida. Estos problemas los ha llevado a la deserción escolar en años anteriores o en otros casos a la promoción escolar sin haber desarrollado las competencias escriturales básicas.

Si persiste esta situación, se corre el riesgo de incrementar cada vez más los índices de analfabetismo y caer en una ruptura cultural, al encontrarse con unos individuos que fueron descartados por la escuela. Para buscar una alternativa didáctica a la enseñanza de la escritura en este tipo de población extra edad, se realiza esta investigación de tipo cualitativo con un enfoque micro etnográfico. El objetivo general es analizar cómo las narrativas autobiográficas como estrategia de intervención en el aula, aportan al conocimiento de sí mismo y permiten fortalecer las habilidades escriturales de los estudiantes del programa "Caminar en secundaria".

Los planteamientos de Miras (2000), Camps (1990; 2000), Zemelman (2010) y Lerner (2001) soportan esta investigación con elementos teórico pedagógicos contundentes que permiten plantear las narrativas autobiográficas como una propuesta pedagógica para crear y recrear al sujeto por medio de la escritura. 
Los estudios realizados hasta ahora, enfatizan en la importancia de las narrativas autobiográficas abordadas en el aula con estudiantes y plantean la escritura de sus vidas más allá un anecdotario, como una relación constante con el pasado en forma reflexiva para construir el futuro mirado en términos de respeto y conciencia. Esto es precisamente lo que plantea la presente investigación de narrativas autobiográficas: motivar a los participantes del proyecto para que se lean, se piensen, se escriban, se transformen y aprendan por medio de las narrativas autobiográficas los conceptos académicos escriturales, y puedan llegar a transformar sus circunstancias personales por medio de la escritura. Dentro de la exploración realizada a nivel local y nacional, se encontraron investigaciones de corte cualitativo donde se emplearon estrategias biográfico-narrativas que presentan la escritura como proceso reflexivo. Todo esto se realiza desde la experiencia de vida, convertida en texto como elemento de intertextualidad para compartir, hacer y rehacer nuevas historias.

\section{Narrativas autobiográficas}

En este grupo se abordan investigaciones donde el planteamiento de la escritura se motiva desde la experiencia y transformación de la realidad a partir de la mirada sensible. Por ejemplo, en la tesis Artesanos de la palabra: una reflexión sobre la experiencia de la lectura y la escritura como prácticas socioculturales y estéticas, Builes (2016) realiza una investigación con docentes en la Normal Superior de Copacabana Antioquia de corte cualitativo, donde se emplean estrategias biográfico-narrativas, a través de las cuales la autora presenta su vida, su ser, su quehacer docente y cómo esto se entreteje mediante la escritura como proceso creativo y reflexivo, así como el de sus pares académicos. Todo esto se realiza desde la experiencia de vida convertida en texto para hacer y rehacer nuevas historias, lo cual permite entender que como lectores y formadores es necesario sensibilizarse y transformarse en un primer momento, para poder tener elementos con los cuales acercarse a los jóvenes, y brindar una experiencia creadora y transformadora en relación con la lectura y la escritura puesta al servicio del conocimiento.

Los resultados de este estudio son de vital importancia para la presente investigación de las narrativas autobiográficas, ya que propicia el encuentro del docente consigo mismo y con el otro para transformarse y transformar. De este modo, se logra la interacción con sus estudiantes haciendo parte de un proceso de investigación-acción-transformación. Para ello, se utilizan instrumentos de recolección de información como los diarios de campo, las bitácoras y las mismas narraciones autobiográficas realizadas por el docente, todas estas herramientas forman parte fundamental de esta intervención. 
Soto (2014) también presenta la tesis La lectura y la escritura como un asunto diferente a la decodificación, donde la escuela debe jugar su papel principal al incorporar estrategias que lleven a los estudiantes a convertirse en escritores de textos, que cumplan funciones sociales en contextos situacionales que le dan sentido a la escritura. Esto a su vez le permite a los estudiantes reconocerse como productores de textos, según lo expresan Lerner (2001) y el Ministerio de Educación Nacional (2008). La autora presenta la escritura de autobiografías como algo más que un anecdotario, esto es, como una relación constante con el pasado en forma reflexiva para construir el futuro, mirado en términos de respeto y conciencia. Esto es precisamente lo que pretende la presente investigación de narrativas autobiográficas, donde la escritura es abordada como una herramienta para recordar, corregir y sensibilizar al otro, a quien lo lee, a quien lo escucha y a sí mismo.

En la tesis Propuesta metodológica para potenciar las competencias escriturales, por medio de la narrativa digital en estudiantes de octavo grado del colegio Alexander Fleming I.E.D, Prieto (2016) aborda el concepto de escritura digital tomando todos los aspectos del ser humano, enfatizando en las narrativas diversas tanto verbales, escritas, no verbales y artísticas, con el uso desde el papel y el lápiz como de herramientas ofimáticas y TIC. La autora se enfoca así en la realidad que vive la sociedad sin dejar de lado la escritura como vehículo que permite formar nuevas realidades y tomar al sujeto como un todo desde el sentir hasta el saber hacer. De esta manera, demuestra la integralidad del sujeto y, con la escritura como un vehículo que conduce al saber y al saberse, confluye con la presente investigación de las narrativas autobiográficas en una búsqueda incesante por transformar la realidad de los sujetos por medio de la escritura.

La propuesta de escritura desde lo social, desde la vivencia cotidiana transformada en texto en la tesis desarrollada en la comunidad de Río Grande en Turbo Antioquia, Las prácticas de la escritura como experiencia sociocultural y su contribución al desarrollo de las capacidades expresivas, Díaz (2015) pretende explicar cómo, por medio de la escritura, se logran desarrollar las capacidades expresivas y apropiarse del entorno cultural a partir de sueños, ideas y anhelos de cambio. Todo esto queda plasmado en su proceso escritural que da cuenta de la forma en que los participantes del proyecto conciben el mundo, en particular su cotidianidad: 1) escritura como reflejo de la literatura para la expresión y transformación del mundo que habitan; 2) la importancia de lo subjetivo, el sentir, la expresión y la emoción como eje inspirador para la escritura. Esta tesis refuerza en gran medida el concepto abordado en la presente investigación, tomando las experiencias cotidianas como el elemento vital para darle fuerza a la escritura desde la expresión del sentimiento. 
En la tesis La escritura autobiográfica: una apuesta pedagógica para la formación de lectores y escritores, Amaya y Pinzón (2015), estudiantes de la Pontificia Universidad Javeriana de Bogotá, enfocan esta investigación en su propia autobiografía. Referencian un proceso vital de escritura que trasciende una propuesta pedagógica didáctica e inspiran a todos a conocerse a sí mismos, a la par que incursionan en el mundo de la escritura, la lectura y la literatura como experiencia de vida llevada a la escritura. De este modo, se persigue en esta investigación la socialización rigurosa del camino de indagación, las prácticas, los procesos y las experiencias asociadas a la lectoescritura. Se genera así un reto y una oportunidad de crecimiento en el proceso escritural desde las experiencias de vida y la cotidianidad para construir los relatos que juegan con la imaginación y se consolidan como referentes importantes para escribir.

\section{Escritura reflexiva}

En este grupo se hallan investigaciones que abordan la escritura de las historias de vida como una alternativa para potenciar la escritura reflexiva desde la experiencia de vida. En la tesis Narrativas de violencia de las y los jóvenes desvinculados de grupos armados al margen de la ley, Iguarán (2011) realiza una investigación enfocada en un grupo de jóvenes de Chiquinquirá y Valledupar. Plantea la reconstrucción del futuro de estos jóvenes por medio de las narraciones autobiográficas y no solo las enmarca como una estrategia de una sola vía, sino que el educador es el primer llamado a confrontar su experiencia de vida, en donde la escritura es el factor primordial para construir un nuevo sujeto y por ende una nueva sociedad. Nuevamente se estructura la historia de vida del docente ligado a la transformación de sujetos en formación y es un referente importante para darle fuerza a esta investigación.

Así mismo, en la tesis La escritura autobiográfica como estrategia de reflexión de las prácticas pedagógicas: análisis de los discursos de los maestros, Páez Gómez y García (2008) realizan un análisis narrativo donde se presenta al docente como el primer sujeto agente transformador de su vida y de sus prácticas de lectura y escritura, tanto en lo que aprende como en lo que enseña. Esta investigación es desarrollada en las instituciones Colegio Monseñor Bernardo Sánchez, el Instituto Icsef y la Universidad San Martín de la ciudad de Bogotá. En esta se concibe la escritura como una estrategia de toma de conciencia y de reflexión aplicada a los docentes como agente transformador, para llevarlo al aula y motivar una experiencia escritural desde la vivencia. Esta investigación realiza aportes precisos para ser aplicados a las narrativas autobiográficas, ya que como docente estoy llamada a transformar mi vida desde la escritura y motivar a los estudiantes para que hagan lo mismo con la suya. 
En la tesis Mi historia de vida en letras: la autobiografía como una estrategia didáctica para mejorar el proceso de composición escrita, Restrepo (2013) realiza la investigación en la Institución Educativa Francisco Miranda del municipio de Medellín que se enfoca en la población adulta de la jornada nocturna. Presenta la narración autobiográfica como un puente entre el alumno y el docente para hacer un proceso de catarsis, y encontrarse con un sujeto que debe transformarse día a día. Hernández et al., como se citó en Restrepo (2013), afirma que "este acto de escribir se realizó a partir de la propia cotidianidad de los estudiantes, al narrar sus propias experiencias, sueños y sentimientos para relacionar lo cognitivo con lo afectivo y con ello alcanzar un autoconocimiento" (p. 43). Para sustentar la importancia que tiene que el sujeto pueda leerse, pensarse y transformarse a través de la escritura de su cotidianidad, se enfatiza que el trabajo autobiográfico potencia los aspectos metacognitivos y mira al sujeto como un agente que no solo recibe información, si no que la interioriza, transforma su pensamiento y realidad.

Por otro lado, esta investigación contextualiza el concepto de competencia, planteado en los lineamientos curriculares como la capacidad con que un sujeto cuenta para escribir su historia y aprender de ella (Ministerio de Educación Nacional, 2008), enfocando el lenguaje en las cuatro habilidades básicas: hablar, escuchar, leer y escribir. La maestra retoma los planteamientos de Freire (2009), donde expone que el individuo debe estar en constante diálogo con el otro, y estar sujeto a incontables revisiones para pensarse y transformarse. Desde la investigación de las narrativas autobiográficas y aplicando los planteamientos de la maestra Restrepo, es posible encontrarse consigo mismo y con el otro desde la escritura de las historias de vida.

Asimimo, Morales y Serrano (2018) presentan en el artículo "La escritura autobiográfica de jóvenes y adultos en educación superior" la formación de los sujetos a partir de la escritura autobiográfica, en los cuales los participantes han plasmado sus historias en diarios, reflexiones, narrativas orales y descripción de escenas de la vida cotidiana con momentos vinculados a saberes y aprendizajes, reflexionan sobre ellos mismos, teniendo como base fundamental para este proceso la escritura. La realidad para leerse, pensarse, escribirse es tomada desde lo cotidiano y se hace academia. Cada una de las anteriores investigaciones confluyen con la propuesta de las narrativas autobiográficas, ya que son una apuesta diferente no solo para promover las prácticas de escritura, sino para transformar al sujeto por medio de una escritura reflexiva de sus vivencias. Es de resaltar que en su mayoría son investigaciones desarrolladas con poblaciones que presentan unas características similares a los estudiantes del programa "Caminar en secundaria" y ello da una luz de esperanza para que la presente investigación sea una guía para futuros trabajos que propendan por mejorar la 
calidad de vida de los sujetos desde las prácticas de escritura, vista más allá de lo académico y con un doble propósito: formar en el ser y en el saber.

\section{Metodología}

Para la ejecución de la presente investigación, se partió de un enfoque cualitativo y un diseño micro etnográfico, se focalizó a través de la observación e interpretación de fenómenos presentados en el grupo a intervenir, para motivar las prácticas de escritura y el fortalecimiento del ser desde las narrativas de los estudiantes, su experiencia de vida, los aspectos del discurso y los juicios de valor que hacen ellos mismos sobre sus realidades. Para este estudio se tomaron diecisiete estudiantes, cinco hombres y doce mujeres al azar del grupo sexto cinco, conformado por veinticinco estudiantes, pertenecientes al programa "Caminar en secundaria" de la institución educativa Ángela Restrepo Moreno; sus edades oscilan entre 13 y 16 años, los cuales presentan unas prácticas de escritura deficiente y unas condiciones familiares y sociales que los ponen en constante riesgo de deserción escolar.

Los datos para este estudio se recopilaron mediante el reconocimiento del grupo de estudio, el diálogo y la observación de su contexto, necesidades e intereses, para diseñar el plan de trabajo. Asimismo, fue necesaria una revisión documental (mallas curriculares, planes de área, DBA y la propuesta del Ministerio de Educación "Caminar en secundaria"), para hallar un punto de encuentro entre estos y la propuesta de investigación. Desde la aplicación de la estrategia metodológica propuesta, se eligen y analizan las categorías inmersas en el problema de investigación y el objetivo general: analizar cómo las narrativas autobiográficas como estrategia de intervención en el aula aportan al conocimiento de sí mismo y permiten fortalecer las habilidades escriturales de los estudiantes elegidos. Las narrativas autobiográficas, la escritura reflexiva, pedagogía y sujeto son categorías que durante el desarrollo de la investigación se observaron, afianzando en gran medida los postulados sobre los cuales se sustenta este trabajo. En la figura 1 se observa la ruta metodológica abordada.

Antes de aplicar la prueba diagnóstica se realizaron actividades de motivación y sensibilización, entre ellas lecturas de biografías de personajes reconocidos y sus docentes más cercanos, seguidamente se aplicó la prueba inicial que consistió en realizar una biografía de un personaje cercano utilizando todos los conocimientos sobre su vida. Dentro de la prueba se miden aspectos escriturales generales: coherencia global, fluidez escritural, cohesión y ortografía. Los estudiantes participantes construyeron una biografía de sus madres. 


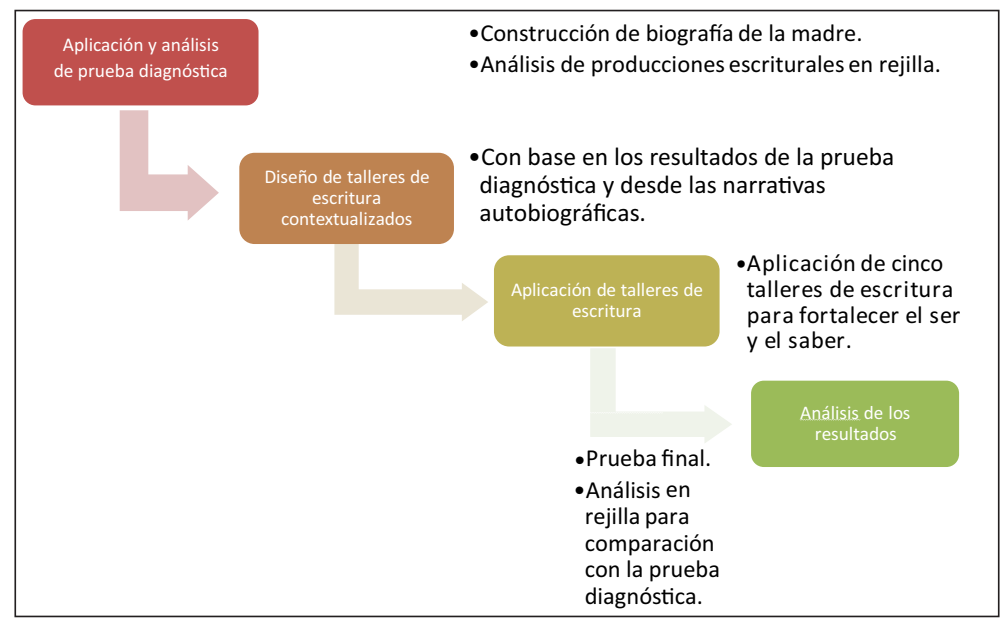

Figura 1. Ruta metodológica "Acción transformadora".

Fuente: elaboración propia basada en la metodología de la investigación.

Durante la actividad se analizaron aspectos escriturales tales como: motivación, ortografía, escritura y reescritura del texto. Asimismo, se evidencia el vínculo afectivo con la persona seleccionada. Luego de analizados los resultados de dicha prueba se procede a diseñar los talleres de escritura (cinco), teniendo en cuenta los aspectos escriturales arrojados como débiles en la prueba diagnóstica. Las actividades de los talleres de escritura estuvieron enfocados a la sensibilización y motivación de la escritura y fortalecimiento del ser, para ello se adaptaron los contenidos al contexto de los estudiantes y a las competencias escriturales del grado. El producto escritural de cada taller se convierte en una puerta para entrar a la siguiente sesión; cada una de ellas es registrada en un diario de campo, el cual es un instrumento para obtener los hallazgos y las categorías emergentes de la investigación.

El taller número uno llamado "Letras que dan vida" se distribuye en dos sesiones. Como punto de partida la biografía, se elige un personaje que les llame la atención, no solo desde su quehacer si no desde su ser, donde se perciben los valores que enmarcan la vida de los estudiantes. Dentro del desarrollo de este se propone un trabajo individual y en equipo con actividades de oralidad, escucha y escritura que se manifestaron cuando, entre ellos mismos reconstruían la historia del personaje. Mediante la producción de los textos los estudiantes pueden leerse, pensarse, escribirse, aprender y fortalecer su auto-conocimiento cuando, al identificarse con el personaje elegido, descubren valores en común y situaciones familiares y sociales que enmarcaron la vida de este como las drogas, o la disciplina que deben desarrollar para llegar hasta el lugar donde actualmente se encuentran. En algunos casos rechazan comportamientos y actitudes del personaje que no desean tener, diciendo "por qué no lo desean". 
En la sesión número dos del taller uno, se propone la construcción de la biografía de un personaje elegido, se sugieren cantantes, actores, entre otros, pero es el mismo estudiante quien elige su personaje. El objetivo es potenciar la escritura reflexiva mediante el análisis de situaciones cotidianas, iniciando por la oralidad, pasando por la escritura y llegando nuevamente a la oralidad y la escucha activa.

El taller de escritura número dos "Tu imagen, mis valores" propone desarrollar un texto reflexivo utilizando conocimientos previos donde se especifica qué valores tiene el personaje elegido y de qué manera los ha vivido el estudiante. Finalmente, en dos párrafos y con sus propias palabras utilizando los conectores, los estudiantesl, realizan una presentación del personaje donde incluyen sus apreciaciones tanto generales como personales. Dentro de los valores destacados por los estudiantes está la solidaridad, la honestidad, la amistad y perseverancia. Los estudiantes son puntuales al describir cómo vivieron esos valores en el pasado, en cuanto a la presentación del personaje tienen la claridad suficiente para argumentar los motivos por los cuales se reconocen con ellos.

Dentro de las producciones se evidencia el placer de escribir sobre sus personajes. Las vivencias y experiencias ajenas aportan al reconocimiento de sí mismos, nos creamos y recreamos desde lo que el otro representa, se convierte en ese espejo en el que nos podemos reflejar con la esperanza de ser mejores.

En cuanto al taller de escritura número tres "Me conozco, reconozco y admiro", cada estudiante construye su autobiografía utilizando los conceptos estudiados en las sesiones anteriores. Para ello, abordan los aspectos pasados, presentes y futuros de su vida, lo que les permite el reconocimiento desde la individualidad cuando se definieron como lo que son y lo que el otro piensa de ellos uniendo hilos conductores por medio de la escritura reflexiva. Lo interesante de este ejercicio radica en que los participantes se visualizan en sus escritos como personas de bien, libres de las circunstancias actuales y ayudando a sus familias.

En la sesión número dos de este taller de escritura, se realiza la socialización de las autobiografías de los estudiantes. Se pasa del pensamiento reflexivo a la escritura y luego la oralidad permite fortalecer el reconocimiento de los aspectos positivos en cada uno de los estudiantes, incluso en aquellos que no leyeron sus producciones u omitieron aspectos; se expresan emociones y sentimientos conocidos y desconocidos para ellos mismos. La producción de sus historias son textos originales, en ellas se reflexiona sobre vivencias y aprendizajes y de una forma sencilla se realizan procesos de catarsis.

En el taller de escritura cuatro "Me visualizo, proyecto y valoro", cada estudiante participante eligió dos objetos representativos en su vida y dos valores 
que se relacionan con esos objetos (manillas, anillos, celular, entre otros). Seguidamente explica y graba en su dispositivo móvil cómo viven o vivieron esos valores en su vida; luego transcribieron en sus cuadernos el texto tal cual quedó en el audio y se realiza la corrección del texto escrito tomado del audio. Se asocian objetos como manillas con amistad, anillo con amor propio, celular con alegría, entre otros. La palabra hablada y luego escrita hace presencia en este taller. Sin embargo, algunos participantes del grupo perdieron la espontaneidad, la fluidez de las emociones y el redireccionamiento del pensamiento. Se mezcla pensamiento, reflexión, oralidad, escucha y escritura cuando los participantes piensan en ese objeto y lo relacionan con un valor reflexionando sobre su importancia para luego oralizarlo, escribirlo y reescribirlo.

En el desarrollo del taller de escritura cinco se retoman elementos de las anteriores intervenciones para continuar motivando y transformando las prácticas escriturales. "Una imagen hecha sentimiento y palabras", es el nombre del taller donde cada estudiante participante tomó el nombre de su madre y lo escribió de forma creativa en la mitad de una hoja, resaltando cuatro valores que la representan. De los valores resaltados en la madre, cada estudiante selecciona dos de ellos, los cuales considera que posee o desea tener. Seguidamente explica cómo vive o desea vivir esos valores seleccionados. Durante el desarrollo de esta práctica se trabaja el pensamiento reflexivo cuando resaltan en sus madres cualidades o actitudes que consideran son importantes. Se pasa del texto escrito a la oralidad, lo que permite afianzar ese concepto de valor no solo en ellos mismos si no en sus contextos.

Consecutivamente y para dar cumplimiento al objetivo de evaluar los resultados obtenidos en las prácticas escriturales, se aplica la prueba final donde nuevamente cada estudiante elabora la biografía de su madre utilizando los conceptos aprendidos. Para el análisis de esta evaluación se tomó la rúbrica con la que se mide la prueba diagnóstica. Los resultados obtenidos mediante la observación participante y la prueba final permiten evidenciar más que modificaciones en las prácticas de escritura, cambios en las actitudes y formas de ver la vida de los estudiantes, aspectos que aportaron a la validez de la investigación.

A continuación, se presentan las categorías de análisis planteadas en la investigación y evidenciadas durante su desarrollo.

\section{Narrativas autobiográficas}

Durante la aplicación de los talleres de escritura permanentemente se desarrolló la escritura reflexiva. En primera instancia y sorprendidos por la intrepidez de los personajes, los estudiantes se dejaron obnubilar y veían en ellos un modelo a seguir. Posteriormente y al realizar una lectura más reflexiva, razonaron sobre 
los antivalores de los personajes, llegando a la conclusión de que no todo lo que hacían era un ejemplo de vida. A través de esta dinámica, los estudiantes lograron mirarse en el otro para conocerse respetando su ser y sentir, para poder ser el otro del otro y ser reconocido.

De acuerdo con Lerner (2001), la historia personal de cada uno es traspasada por esta experiencia de conocimiento y expresión. No consiste solo en repetir una historia social o cultural, sino trascender desde una trasformación orientada desde la escuela como punto de encuentro y eje articulador de experiencias cognoscitivas y sensitivas, que impactan el ser y se extiende a su entorno familiar y social para afectarlo positivamente. Pasando por procesos consientes e inconscientes el sujeto se vuelve producto de historias cuando evoca su pasado, cuando se reconoce en su presente y se proyecta en su futuro. Expresiones como "yo también estuve en un internado" demuestran que existe una afectación del otro a través de las historias escuchadas que ha vivido, llevando así a realizar una revisión de conciencia y una toma de decisiones motivada por la vivencia del otro.

Asimismo, se evidenciaron procesos de concientización y trasformación en los estudiantes participantes, sus formas de pensar y actuar cuando se manifiesta un evento involuntario que ha marcado la vida y que aún continúa afectando, pero del cual se puede elegir salir y dejar atrás. En esta fase de la investigación se evidenció cómo mediante la escucha de las otras historias, se potencia el ser, la auto curación de las emociones y se fortalece la escritura reflexiva. De igual manera como investigadora, la observación participante me permitió adentrarme en cada una de las historias leídas y escuchadas para llevarlas al diario de campo, y posteriormente analizar los hallazgos encontrados para dar respuesta a la pregunta de investigación.

\section{Escritura reflexiva}

Cuando el sujeto inicia el reconocimiento de sí mismo, empieza un recorrido desde su saber(se), leer(se), escribir(se), para llegar a la movilización del pensamiento desde la escritura reflexiva; las historias de vida permitieron el encuentro consigo mismo por medio de la lectura y la escritura. En esta categoría se incluyó el desarrollo de las habilidades escriturales sobre sus cotidianidades para llegar al reconocimiento del ser y el saber:

Lo necesario es hacer de la escuela un ámbito donde lectura y escritura sean prácticas vivas y vitales, donde leer y escribir sean instrumentos poderosos que permitan repensar el mundo y reorganizar el propio pensamiento, donde interpretar y producir textos sean derechos que es legítimo ejercer y responsabilidades que es necesario asumir. (Lerner, 2001, p. 26)

Cuando los estudiantes socializaron sus producciones autobiográficas se evidenció la escritura reflexiva, ya que pasó del texto académico al conocimiento 
de sí mismo. Fue un encuentro con el yo interior que permitió tomar una posición clara por encima de unas circunstancias, independiente de las vivencias de sus padres, incluso de ellos mismos; permitió llegar a la escritura como una herramienta para tomar conciencia, donde el sujeto se conoce, reconoce y toma una posición desde un sentir y un actuar para regular su pensamiento a través de la oralidad y la escritura. Los estudiantes se cuestionaron con respecto a actitudes de sus madres, y se preguntaron si son valores solo porque los exigen en ellos, pero no los viven. La escritura inicialmente tomó forma en el pensamiento pasando a la oralidad y luego a la escritura, es evidente que la historia del otro permea la vida del individuo.

\section{Pedagogía y sujeto}

La estrategia del taller de escritura permitió el goce y el disfrute y a su vez fortaleció las habilidades escriturales de los estudiantes. Fueron de vital importancia cada una de las sesiones tanto grupales como individuales, ya que en cada una de ellas se alcanzó la transformación de las practicas escriturales, las cuales fortalecieron el desarrollo cognitivo y la competencia escritural. Según Núñez, Téllez, y Castellanos (2017) "los materiales desarrollados por los docentes encajan en la categoría de materiales contextualizados que están ligados al contexto ya que responden a las necesidades locales" (p. 34). En este sentido, los talleres de escritura utilizados para el fortalecimiento del sujeto fueron un elemento pedagógico que aportaron al conocimiento académico y de sí mismo

Dentro del desarrollo de la investigación surgieron unas categorías que si bien es cierto no se consideraron como relevantes al inicio de esta, fueron de vital importancia y aportaron en gran medida para cumplir el objetivo general. Estas categorías permitieron que los estudiantes fortalecieran sus ideas por medio de discusiones y escucha activa de los puntos de vista del otro. Goodman (1982) argumenta que es necesario generar espacios de construcción grupal, permitir escuchar al otro, respetar puntos de vista y compartir experiencias para llegar a la construcción de significados a partir de su propósito de lectura y los diversos esquemas y estrategias cognitivos que ponen en juego al momento de leer, como lo son la predicción, la inferencia, la verificación y la corrección.

Este aspecto favoreció en gran medida la producción escritural, puesto que generó confianza y empatía al escuchar las historias de vida. Asimismo, permitió la interacción entre los estudiantes y los ayudó a comprender mejor los temas. Algunos tomaron inicialmente el papel de observadores y finalmente dieron cuenta de la comprensión de los temas realizando exposiciones con gran propiedad de los personajes elegidos, lo cual demostró que hay otras formas 
de llegar a la producción escritural: el trabajo en equipo potencia la escucha, la observación y la lectura que se hace del otro, influyen notablemente en la formación y transformación del sujeto. La socialización de las autobiografías que se realizaron de forma libre y espontánea permitió a quienes escucharon luego producir escritura. Asimismo, se facilitó un pensamiento reflexivo en quienes aparentemente eran espectadores. La oralidad jugó un papel fundamental en el desarrollo de este taller, ya que lo que no se logró de forma escritural se desarrolló desde la oralidad. La construcción y reconstrucción del concepto de valor se realizó de forma grupal, llevando a reflexionar sobre ciertas actitudes y comportamientos que poseen las personas y que no por ser famosas están bien hechas.

Dentro de los procesos de la escritura basados en las narrativas autobiográficas se puso en juego la capacidad de reflexionar sobre su entorno y lograr modificarlo diariamente, no solo en el papel, sino dentro de una realidad. Cuando los estudiantes escribieron cómo se veían en un futuro, la escritura permeó el ser para llevarlo al hacer. Se creó y recreo la existencia, a la vez que se abrió un espacio a la rigurosidad del texto donde no solo se escribe, sino que este acto se planifica, reflexiona y se cuestiona como forma de autocrítica y producción sensible. Con respecto a ello, Velasco (2010) afirma:

Nos construimos sobre narraciones, somos a partir de los relatos. Ello es lo que asegura nuestra identidad a lo largo de lo cambiante de nuestras vidas. Como personajes de un relato, nuestra identidad es dinámica: el relato construye la identidad del personaje, que podemos llamar su identidad narrativa, al construir la identidad dinámica propia de la historia contada. La identidad de la historia forja la del personaje. (p. 23)

De ahí la importancia de conocernos como sujetos, reconocernos y estar en constante proceso de cambio: nos leemos, nos pensamos, nos escribimos, nos transformamos y aprendemos. Tomar las narrativas autobiográficas como punto de partida nos lleva a una conciencia y dominio propio, y por ende a la trasformación del sujeto. El relato autobiográfico del otro se convierte en un referente de partida donde el proceso reflexivo elaborado por el otro se trasforma en un yo, llevando a sentir y proyectar por medio de la escucha una nueva forma de ver y sentir la vida, y es precisamente la escuela quien debe promover que se lea y se escriba desde el ser y el sentir.

\section{Resultados}

Luego de aplicada la estrategia metodológica planteada desde la prueba diagnóstica, los talleres de escritura y la prueba final, se evidenciaron los siguientes resultados analizados a la luz de los objetivos propuestos. 
1. Respecto al objetivo de diagnosticar los niveles de escritura del grupo seleccionado, se evidencia un alto porcentaje en la redundancia en las ideas, poca cohesión, falta de fluidez y coherencia, además de la omisión de algunas palabras en sus escritos. Contrastados luego los resultados iniciales y finales en cuanto a la coherencia global, se comprobó un cambio positivo en la presentación de las ideas en forma lógica y secuencial, ya que en la primera prueba el $18 \%$ de los estudiantes alcanza una coherencia en sus escritos, mientras que en la segunda prueba el 59 \% de los estudiantes lo logra.

2. En cuanto a la fluidez escritural, al realizar la comparación de los resultados iniciales y finales, también se nota un cambio positivo, se pasó del $30 \%$ al 59 \% . Lo mismo ocurrió con la cohesión de los escritos, en la prueba inicial el $30 \%$ de los estudiantes son coherentes en sus producciones y en la segunda prueba es evidente la mejoría pasando al 70 \%. En cuanto a las omisiones y repeticiones de las palabras, se evidencia una mejoría aunque no muy notoria, ya que es una de las mayores dificultades de estos estudiantes. En la prueba inicial, el $12 \%$ de ellos, que equivale a dos estudiantes, no presenta falencias en la producción y en la prueba final aumentó a 18 \%, lo que equivale a tres estudiantes. En cuanto a la variedad y economía del lenguaje se apreció un aumento favorable al nombrar las cosas por su nombre y expresar las ideas en forma clara y concisa. El mayor logro se observó en la construcción del ser, ya que se expresaron y liberaron emociones, razonando sobre ideas preconcebidas de actitudes que en algún momento dañaron a los estudiantes o incluso que en la actualidad hacen parte de su vida. A continuación, se anexan las tablas 1, 2, 3 ,4, 5 y 6 donde se describen los resultados de las pruebas diagnósticas y de la prueba final.

Tabla 1. Coherencia global

\begin{tabular}{|l|c|c|c|c|}
\hline Aspectos escriturales & $\begin{array}{c}\text { Valoración } \\
\text { inicial }\end{array}$ & Observaciones. & $\begin{array}{c}\text { Valoración } \\
\text { final }\end{array}$ & Observaciones \\
\hline $\begin{array}{l}\text { No coherente. Las } \\
\text { ideas no guardan re- } \\
\text { lación lógica de prin- } \\
\text { cipio a fin. }\end{array}$ & 3 & $\begin{array}{l}\text { El 18\% de los estu- } \\
\text { diantes no presen- } \\
\text { tan coherencia en } \\
\text { sus escritos. }\end{array}$ & 2 & $\begin{array}{l}\text { El 12\% de los estudian- } \\
\text { tes no presentó cohe- } \\
\text { rencia en sus escritos. }\end{array}$ \\
\hline $\begin{array}{l}\text { Coherencia parcial. } \\
\text { Existe una relación ló- } \\
\text { gica entre algunas de } \\
\text { las ideas. }\end{array}$ & 11 & $\begin{array}{l}\text { El } 65 \% \text { de los es- } \\
\text { critos presenta una } \\
\text { coherencia parcial. }\end{array}$ & 5 & $\begin{array}{l}\text { El 29\% de los estudian- } \\
\text { tes presentaron una co- } \\
\text { herencia parcial en sus } \\
\text { escritos. }\end{array}$ \\
\hline $\begin{array}{l}\text { Coherente. Las ideas } \\
\text { presentan un orden } \\
\text { lógico y secuencial. }\end{array}$ & 3 & $\begin{array}{l}\text { El } 18 \% \text { de las pro- } \\
\text { ducciones son co- } \\
\text { herentes. }\end{array}$ & 10 & $\begin{array}{l}\text { El } 59 \% \text { de los estudian- } \\
\text { tes presentaron un or- } \\
\text { den lógico y secuencial } \\
\text { en sus textos. }\end{array}$ \\
\hline
\end{tabular}

Fuente: elaboración propia. 
Tabla 2. Fluidez escritural

\begin{tabular}{|l|c|c|c|c|}
\hline \multicolumn{1}{|c|}{ Aspectos escriturales } & $\begin{array}{c}\text { Valoración } \\
\text { inicial }\end{array}$ & observaciones & $\begin{array}{c}\text { Valoración } \\
\text { final }\end{array}$ & Observaciones \\
\hline $\begin{array}{l}\text { No fluido. Ausencia de } \\
\text { ideas, lo cual se manifies- } \\
\text { ta en la calidad de las ideas } \\
\text { y en la extensión del texto. }\end{array}$ & 5 & $\begin{array}{l}\text { El 30\% de los es- } \\
\text { critos presenta un } \\
\text { texto fluido. }\end{array}$ & 5 & $\begin{array}{l}\text { El 30\% de los } \\
\text { estudiantes pre- } \\
\text { senta un texto } \\
\text { fluido. }\end{array}$ \\
\hline $\begin{array}{l}\text { Más o menos fluido. Pocas } \\
\text { ideas tanto en calidad como } \\
\text { en extensión. }\end{array}$ & 7 & $\begin{array}{l}\text { El } 40 \% \text { más o me- } \\
\text { nos fluido. }\end{array}$ & 2 & $\begin{array}{l}\text { El } 12 \% \text { de los } \\
\text { textos de los es- } \\
\text { tudiantes fueron } \\
\text { más o menos } \\
\text { fluidos. }\end{array}$ \\
\hline $\begin{array}{l}\text { Fluido. Riqueza de ideas, lo } \\
\text { cual se manifiesta en la ca- } \\
\text { lidad de las ideas y en la ex- } \\
\text { tensión. }\end{array}$ & 5 & $\begin{array}{l}\text { El30\% presenta ri- } \\
\text { queza en las ideas } \\
\text { siendo fluido. }\end{array}$ & 10 & $\begin{array}{l}\text { El 59\% de los } \\
\text { estudiantes pre- } \\
\text { sento una rique- } \\
\text { za de ideas. }\end{array}$ \\
\hline
\end{tabular}

Fuente: elaboración propia.

Tabla 3. Cohesión

\begin{tabular}{|l|c|c|c|c|}
\hline \multicolumn{1}{|c|}{$\begin{array}{c}\text { Aspectos } \\
\text { escriturales }\end{array}$} & $\begin{array}{c}\text { Valoración } \\
\text { inicial }\end{array}$ & Observaciones & $\begin{array}{c}\text { Valoración } \\
\text { final }\end{array}$ & Observaciones \\
\hline $\begin{array}{l}\text { Omite o utiliza in- } \\
\text { discriminadamen- } \\
\text { te los signos de } \\
\text { puntuación. }\end{array}$ & 12 & $\begin{array}{l}\text { El 70\% de los escritos } \\
\text { utiliza adecuadamen- } \\
\text { te los signos de pun- } \\
\text { tuación, no hay sepa- } \\
\text { ración de párrafos. }\end{array}$ & 5 & $\begin{array}{l}\text { El 30 \% de los estu- } \\
\text { diantes omiten o uti- } \\
\text { lizan indiscretamente } \\
\text { los signos de puntua- } \\
\text { ción. }\end{array}$ \\
\hline $\begin{array}{l}\text { Utiliza los signos } \\
\text { de puntuación par- } \\
\text { cialmente. }\end{array}$ & 5 & $\begin{array}{l}\text { El 30 \% de los escri- } \\
\text { tos presenta signos } \\
\text { de puntuación, uso } \\
\text { de mayúsculas y dis- } \\
\text { tribución de párrafos. }\end{array}$ & 12 & $\begin{array}{l}\text { El 70 \% de los estu- } \\
\text { diantes utiliza par- } \\
\text { cialmente los signos } \\
\text { de puntuación en sus } \\
\text { escritos. }\end{array}$ \\
\hline $\begin{array}{l}\text { Utiliza los signos } \\
\text { de puntuación ade- } \\
\text { cuadamente. }\end{array}$ & 0 & & & \\
\hline
\end{tabular}

Fuente: elaboración propia.

Tabla 4. Escritura correcta de las palabras

\begin{tabular}{|c|c|c|c|c|}
\hline \multicolumn{1}{|c|}{$\begin{array}{c}\text { Aspectos } \\
\text { escriturales }\end{array}$} & $\begin{array}{c}\text { Valoración } \\
\text { inicial }\end{array}$ & Observaciones & $\begin{array}{c}\text { Valoración } \\
\text { final }\end{array}$ & Observaciones \\
\hline $\begin{array}{l}\text { Presenta en su escri- } \\
\text { to omisiones, repeti- } \\
\text { ciones, inversiones o } \\
\text { sustituciones de le- } \\
\text { tras o palabras. }\end{array}$ & 15 & $\begin{array}{l}\text { El 88 \% de los es- } \\
\text { critos presenta } \\
\text { omisiones y una } \\
\text { escritura defi- } \\
\text { ciente. }\end{array}$ & 14 & $\begin{array}{l}\text { El 82 \% de los estu- } \\
\text { diantes presenta en } \\
\text { sus escritos repeticio- } \\
\text { nes, omisiones y una } \\
\text { escritura deficiente. }\end{array}$ \\
\hline
\end{tabular}


Narrativas autobiográficas, una estrategia pedagógica para transformar el ser desde la escritura

\begin{tabular}{|l|c|c|c|c|}
\hline \multicolumn{1}{|c|}{$\begin{array}{c}\text { Aspectos } \\
\text { escriturales }\end{array}$} & $\begin{array}{c}\text { Valoración } \\
\text { inicial }\end{array}$ & Observaciones & $\begin{array}{c}\text { Valoración } \\
\text { final }\end{array}$ & Observaciones \\
\hline $\begin{array}{l}\text { No presenta en su es- } \\
\text { crito omisiones, repe- } \\
\text { ticiones, inversiones } \\
\text { o sustituciones de le- } \\
\text { tras o palabras. }\end{array}$ & 2 & $\begin{array}{c}\text { El 12\% presenta } \\
\text { una escritura más } \\
\text { o menos correcta, } \\
\text { sin omisiones o } \\
\text { repeticiones. }\end{array}$ & 3 & $\begin{array}{l}\text { El 18 \% de los estu- } \\
\text { diantes presenta una } \\
\text { escritura más o me- } \\
\text { nos correcta, sin omi- } \\
\text { siones o repeticiones. }\end{array}$ \\
\hline
\end{tabular}

Fuente: elaboración propia.

Tabla 5. Economía en el lenguaje

\begin{tabular}{|l|c|c|c|c|}
\hline \multicolumn{1}{|c|}{$\begin{array}{c}\text { Aspectos } \\
\text { escriturales }\end{array}$} & $\begin{array}{c}\text { Valoración } \\
\text { inicial }\end{array}$ & Observaciones & $\begin{array}{c}\text { Valoración } \\
\text { final }\end{array}$ & Observaciones \\
\hline $\begin{array}{l}\text { Presenta redun- } \\
\text { dancia de ideas y } \\
\text { repetición de pa- } \\
\text { labras. }\end{array}$ & 16 & $\begin{array}{l}\text { El 94 \% de los es- } \\
\text { critos son redun- } \\
\text { dantes. }\end{array}$ & 4 & $\begin{array}{l}\text { El 24 \% de los estudian- } \\
\text { tes presenta redundan- } \\
\text { cia de ideas y repeticio- } \\
\text { nes de palabras. }\end{array}$ \\
\hline $\begin{array}{l}\text { Expresa las ideas } \\
\text { en forma direc- } \\
\text { ta y concisa solo } \\
\text { en una parte del } \\
\text { texto. }\end{array}$ & 1 & $\begin{array}{l}\text { El 6 \% expresa en } \\
\text { forma directa y flui- } \\
\text { da las ideas. }\end{array}$ & 3 & $\begin{array}{l}\text { El 18\% de los estudian- } \\
\text { tes expresa en forma di- } \\
\text { recta y fluida las ideas. }\end{array}$ \\
\hline $\begin{array}{l}\text { Expresa las ideas } \\
\text { en forma directa } \\
\text { y concisa. }\end{array}$ & & 9 & $\begin{array}{l}\text { El 53\% de los estudian- } \\
\text { tes expresa las ideas en } \\
\text { forma directa y concisa. }\end{array}$ \\
\hline
\end{tabular}

Fuente: elaboración propia.

Tabla 6. Variedad en el lenguaje

\begin{tabular}{|l|c|l|c|c|}
\hline \multicolumn{1}{|c|}{$\begin{array}{c}\text { Aspectos } \\
\text { escriturales }\end{array}$} & $\begin{array}{c}\text { Valoración } \\
\text { inicial }\end{array}$ & Observaciones & $\begin{array}{c}\text { Valoración } \\
\text { final }\end{array}$ & Observaciones \\
\hline $\begin{array}{l}\text { No denomina las } \\
\text { cosas por su nom- } \\
\text { bre }\end{array}$ & 1 & $\begin{array}{l}\text { El 6 \% de los escri- } \\
\text { tos denomina las } \\
\text { cosas por sus nom- } \\
\text { bres }\end{array}$ & 6 & $\begin{array}{l}\text { El 35 \% de los es- } \\
\text { tudiantes no nom- } \\
\text { bra las cosas por su } \\
\text { nombre }\end{array}$ \\
\hline $\begin{array}{l}\text { Algunas veces } \\
\text { nombra las cosas } \\
\text { por su nombre }\end{array}$ & 7 & $\begin{array}{l}\text { El } 41 \% \text { presenta un } \\
\text { lenguaje confuso }\end{array}$ & 6 & $\begin{array}{l}\text { El } 65 \% \text { de los es- } \\
\text { tudiantes denomi- } \\
\text { na las cosas por su } \\
\text { nombre }\end{array}$ \\
\hline $\begin{array}{l}\text { Nombra correcta- } \\
\text { mente las cosas } \\
\text { por su nombre }\end{array}$ & 9 & $\begin{array}{l}\text { El 53\% de los escri- } \\
\text { tos narra de forma } \\
\text { concisa, llaman- } \\
\text { do las cosas por su } \\
\text { nombre }\end{array}$ & 11 & \\
\hline
\end{tabular}

Fuente: elaboración propia a partir Hurtado, Restrepo y Herrera (2005). 
3. En lo relacionado con el segundo y tercer objetivo de la investigación, elaborar y aplicar talleres de escritura que motivaran las prácticas de escritura, el resultado fue satisfactorio. De los cinco talleres de escritura programados se ejecutan el $100 \%$, las actividades contextualizadas desde los gustos e intereses de los estudiantes fueron de vital importancia para fortalecer la escritura. Esto permite el reconocimiento del ser hasta llegar al sujeto, movilizando el pensamiento cuando en sus escritos manifestaron los motivos por los que admiraban al personaje. Sin embargo, destacan lo que no desean tener de él y lo que no comparten, reflexionan sobre lo que consideran un valor y a su vez se identifican con él, manifestando el placer de escribir sobre el otro desde el reconocimiento de sí mismo, ya que los seres humanos nos creamos y recreamos a partir de lo que el otro representa.

4. Los participantes tambien logran leerse y escribirse al identificarse con los valores que resaltan de sus madres, pensarse y escribirse cuando toman una posición clara y argumentada de lo que no consideran adecuado, aunque se refieran a los comportamientos de su madre; transformarse y aprender cuando escriben es el deseo de una nueva vida independiente de sus circunstancias actuales. El trabajo grupal canaliza y fortalece la escucha y la observación que se hace del otro, influyendo notablemente en la formación y transformación del sujeto, cuando luego en sus escritos plasman muchas de las ideas escuchadas, haciéndolas parte de su escritura.

5. Desde la oralidad de sus narrativas autobiográficas se producen textos originales. Al leerlos, hay una trasformación de pensamiento cuando argumentan que son seres valiosos, que podrán lograr cualquier sueño y que no por haber cometido errores son seres sin valor. Por otro lado, se destaca que por medio del trabajo colaborativo se fortalece la escritura, ya que los estudiantes que no tenían la iniciativa de realizar actividades individuales finalmente aportaron al desarrollo del producto final y dieron cuenta de todo el proceso, a su vez favoreció comportamientos relacionados con los procesos de escritura. También se evidencia que los estudiantes entablan diálogos sobre el tema planteado, por ende, se amplían los horizontes de pensamiento y se lleva a la escritura.

\section{Conclusiones}

Abordar el tema de las narrativas autobiográficas como una estrategia que permita motivar las prácticas de escritura, el conocimiento de sí mismo y de su entorno en una población extra edad, permite comprobar que desde la escuela es preciso una búsqueda permanente de estrategias para llevar el conocimiento a cada uno de los individuos involucrados en la educación, siendo el currículo un elemento vivo que permite ser modificado de acuerdo con las necesidades de los actores. 
Las narrativas autobiográficas como estrategia de intervención en el aula permiten la formación de estudiantes productores de textos, ya que los escritos basados en las experiencias de vida de cada uno de los estudiantes participantes fortalece la escritura y moviliza pensamiento. De esta manera se logran potenciar las habilidades escriturales en este tipo de población extra edad y a su vez aportan al conocimiento de sí mismos, ya que cuando se parte de las necesidades e intereses de los sujetos para motivar la escritura, se puede formar estudiantes productores en lugar de reproductores de textos, pues la argumentación está basada en experiencias reales que llevan a la reflexión y potencian su ser y saber.

Con la aplicación de la estrategia planteada desde las narrativas autobiográficas en el aula, se logró vincular la producción escrita a la experiencia de vida, ya que moviliza fibras que motivaron la transformación del pensamiento que, llevado a la escritura, brindan bases conceptuales establecidas que le permiten a los mismos estudiantes un anclaje a la escritura para conocer y conocerse.

Las actividades contextualizadas como las narrativas autobiográficas permiten la sensibilización de los estudiantes, motivan las prácticas de escritura y es posible abrir puertas hacia el conocimiento siempre y cuando sean no abordadas como un anecdotario, sino como una reflexión constante con el pasado y el presente. Es una herramienta para que los jóvenes en condiciones extra edad inicien la construcción de un camino día a día, no solo desde el ser sino hasta el saber. Es importante que desde la misma escuela se dé respuesta a la necesidad de cada uno de los individuos y por medio de la escritura proyectar otra realidad en función de mejorar los procesos de aprendizaje que los lleve a establecer otras formas de comunicarse consigo mismo y con los demás.

Es necesario que los directivos docentes y los docentes realicen una revisión permanente de los currículos sobre todo para poblaciones especiales como los pertenecientes al programa "Caminar en secundaria", para contextualizar y dinamizar los procesos académicos enfocándolos en el ser, ya que la mayoría de los estudiantes de esta población traen consigo un sin número de situaciones que les han impedido continuar sus estudios. Aunque se encuentran dentro del sistema educativo, son más vulnerables que el resto de la población porque se ven incitados a abandonar nuevamente la escuela. Por otro lado, a pesar de que el modelo plantea estrategias de aprendizaje válidas para las características de los estudiantes como el dictado y la transcripción de textos, el análisis de situaciones cotidianas no son las únicas que deben utilizarse, ante todo si se tiene en cuenta la necesidad de innovar en el aula y crear ambientes diferentes que motiven el aprendizaje y formen sujetos que pasen por el ser y el saber desde la contextualización de las prácticas de escritura, para que sea posible leer y escribir en la escuela y trascender a la sociedad que los ha dejado a un lado. 
Las narrativas autobiográficas como una estrategia para motivar las prácticas de escritura promueven la reflexión sobre el ejercicio docente y la transformación que debemos hacer permanentemente en nosotros como sujetos transformados para transformar y contribuir a la formación de una mejor sociedad mediante la movilización del pensamiento. De esta manera, se aporta a la visión de la educación de promover la formación de personas capaces de vivir en sociedad, líderes de su propia vida, comprometidas social y ambientalmente, que ayuden a los demás y lleguen a la transformación individual y comunitaria en eventos cotidianos de lectura y escritura.

Los docentes de los modelos educativos flexibles estamos llamados a estar en una permanente búsqueda para incluir en las prácticas pedagógicas acciones que conlleven a las habilidades de la autonomía escritural. Por medio de ellas, es posible rescatar el valor de aquellos sujetos que no han podido reconocerse y que se encuentran en grado sexto en su mayoría no por desconocimiento académico, sino por sus condiciones sociales y familiares, las cuales les han impedido permanecer en el sistema educativo. Por ello, las narrativas autobiográficas son una apuesta para motivar en esta población la búsqueda de una mejor calidad de vida cimentada desde la escritura.

Como sujetos, estudiantes, docentes, es necesario conocer y escribir nuestra historia de vida. Tenemos un compromiso con nosotros mismos y con nuestras futuras generaciones de inmortalizarnos en el papel, dibujarnos con palabras, sanar nuestro pasado, despojarnos del dolor y las emociones que las acompañan y así tomar mejores decisiones desde el ser, enfocadas desde la escritura reflexiva, ya que ello permite el encuentro consigo mismo y el reconocimiento de valores y cualidades, así como forjar un futuro diferente y una identidad intencionada a la búsqueda de la trascendencia.

Si bien es cierto que las actividades desarrolladas desde las narrativas autobiográficas fortalecen las prácticas de escritura de los estudiantes, y que la estrategia de intervención presentada en esta investigación fue beneficiosa para los estudiantes objeto de estudio, es claro que se requiere profundizar en otros factores que afectan la práctica de la escritura y que aún queda mucho campo por explorar, de modo que se logre llevar la propuesta de las narrativas autobiográficas a las demás áreas del conocimiento como una forma de transversalizar el saber y comprobar cómo a medida que el sujeto se lee y escribe puede acceder con mayor facilidad a otros campos del conocimiento. Estas hipótesis serán comprobadas en la medida que nuevas investigaciones le apuesten a las narrativas autobiográficas como una forma de movilizar pensamiento y fortalecer el aprendizaje 


\section{Agradecimientos}

Este artículo presenta los resultados del trabajo de investigación denominado: Narrativas autobiográficas, una propuesta pedagógica para crear y recrear las prácticas de escritura: me leo, me pienso, me escribo, me transformo y aprendo, realizado durante la Maestría en Educación, en la línea Didáctica de la Lectura y la Escritura de la Universidad de Medellín. La investigación fue orientada por la profesora Luz Adriana Restrepo Calderón, magíster en Docencia de Lenguaje y Educación de la Universidad de Antioquia, y bajo la asesoría del profesor Julio Sampedro Longas, magíster en Estética de la Universidad de Antioquia.

\section{Referencias}

Amaya, E., y Pinzón, P. (2015). La escritura autobiográfica una apuesta pedagógica para la formación de lectores y escritores (tesis de maestría). Bogotá: Pontificia Universidad Javeriana. Recuperado de: http://hdl.handle.net/10554/18694

Builes, L. F. (2016). Artesanos de la palabra: una reflexión sobre la experiencia de la lectura y la escritura como prácticas socioculturales y estéticas (tesis de maestría). Medellín: Universidad de Antioquia. Recuperado de: http://bibliotecadigital.udea.edu.co/dspace/handle/10495/5234

Camps, A. (2000): Motivos para escribir. Textos de Didáctica de la Lengua y de la Literatura, 23, 69-78.

Camps, A. (1990): Modelos de procesos de redacción: Algunas implicaciones para su enseñanza. Infancia y Aprendizaje, 49, 3-21.

Díaz, R. (2015). Las prácticas de la escritura como experiencia sociocultural y su contribución al desarrollo de las capacidades expresivas (tesis de maestría). Turbo, Antioquia: Universidad de Antioquia. Recuperado de: http://bibliotecadigital.udea.edu.co/bitstream/10495/5274/1/RitcelinaDiazA 2015 practicaescriturasociocultural.pdf.

Freire, P. (2009). Pedagogía del compromiso. América Latina y Educación Popular. Barcelona: Hipatia Editorial.

Hurtado, R., Restrepo, L. y Herrera, O. (2005). Escritura reflexiva: una propuesta didáctica para la básica primaria. Medellín: Universidad de Antioquia.

Iguarán, J. L. (2011). Narrativas de violencia de las y los jóvenes desvinculados de grupos armados al margen de la ley (tesis de grado). Bogotá: Pontificia Universidad Javeriana.

Lerner, D. (2001). Leer y escribir en la escuela: lo real, lo posible y lo necesario. Ciudad de México: Fondo de Cultura Económica. Recuperado de: http://formacion.sigeyucatan.gob.mx/formacion/materiales/4/6/d2/p2/2.\%20Leer.y.escribir.en.la.escuela\%20Lerner.pdf

Miras, M. (2000). La escritura reflexiva. Aprender a escribir y aprender acerca de lo que se escribe. Infancia y aprendizaje, 23(1), 65-80.

Morales, J., y Serrano, J. (2018). La escritura autobiográfica de jóvenes y adultos en educación superior. Revista Brasileira de Educação de Jovens e Adultos, 5(10), 77-89.

Núñez, A., Téllez, M., y Castellanos, J. (2017). Materials Development for Teachers' Professional Growth. En A. Núñez, M. Téllez y J. Castellanos (eds.), Materials for the learning of english and teachers professional growth (pp. 19-68). Bogotá: Universidad Externado de Colombia. 
Páez, A., Gómez, C., y García, M. (2008). Escritura autobiográfica como estrategia de reflexión de las prácticas pedagógicas: análisis de los discursos de los maestros [tesis de maestría]. Bogotá: Universidad de la Salle. Recuperado de: http://repository.lasalle.edu.co/bitstream/handle/10185/1454/ T85.08\%20P139e.pdf?sequence=1

Prieto, D. (2016). Propuesta metodológica para potenciar las competencias escriturales, por medio de la narrativa digital en estudiantes de octavo grado del colegio Alexander Fleming I.E.D (tesis de maestría). Bogotá: Universidad Libre. Recuperado de: https://repository.unilibre. edu.co/bitstream/handle/10901/9558/TESIS\%20DAMARIS\%20PRIETO\%20CALDER\%C3\%93N. pdf?sequence $=1 \&$ is Allowed $=$

Restrepo, D. V. (2013). Mi historia de vida en letras: la autobiografía como una estrategia didáctica para mejorar el proceso de composición escrita (tesis de grado). Medellín: Universidad de Antioquia. Recuperado de: http://ayura.udea.edu.co:8080/jspui/bitstream/123456789/1580/1/PA0797. pdf

Restrepo, L. A. (2013). ¿Formar niños productores o reproductores de textos? En R. D. Hurtado Vergara (coord.), Perspectivas contemporáneas sobre la didáctica de la lectura y la escritura en la infancia (pp. 1-16). Medellín, Colombia: Universidad de Antioquia.

Ricoeur, P. (1999). La lectura del tiempo pasado: memoria y olvido. Madrid: Arrecife Producciones.

Soto, J. (2014). Incidencia de una secuencia didáctica basada en las categorías de contexto y situación de comunicación para la producción de textos autobiográficos en estudiantes de grado $4^{\circ}$ y $5^{\circ}$ E.B.P (tesis de maestría). Pereira: Universidad Tecnológica de Pereira. Recuperado de: http://repositorio. utp.edu.co/dspace/bitstream/handle/11059/4083/37241S718.pdf?sequence=1

Velasco, M. F. (2010). Paulo Freire, Paul Ricoeur y la identidad narrativa. Realidad: Revista de Ciencias Sociales y Humanidades, 123, 117-147. Recuperado de: https://doi.org/10.5377/realidad.v0i123.3317

Zemelman Merino, H. (2010). Sujeto y subjetividad: la problemática de las alternativas como construcción posible. Polis, Revista Latino Americana, 27, 1-11. 